\title{
Flipped Learning en prácticas de matemáticas en Ingeniería Electrónica. Una experiencia piloto.
}

\section{Flipped Learning in mathematics lab sessions in an Electronic Engeeniering Degree. A pilot experience.}

\author{
José Tomás Alcalá Nalvaiz ${ }^{1}$, Natalia Boal Sánchez ${ }^{2}, \mathrm{M}^{\mathrm{a}}$ Inmaculada Gómez Ibañez ${ }^{2}$, Sergio Serrano Pastor ${ }^{2}$ \\ jtalcala@unizar.es,nboal@unizar.es, igomez@unizar.es, sserrano@unizar.es \\ ${ }^{2}$ Departamento de Matemática Aplicada \\ Universidad de Zaragoza \\ Zaragoza, España \\ ${ }^{1}$ Departamento de Métodos Estadísticos \\ Universidad de Zaragoza \\ Zaragoza, España
}

\begin{abstract}
Resumen- En este trabajo se resume una experiencia de clase invertida en las sesiones de laboratorio informático de matemáticas en un Grado de Ingeniería Electrónica y Automática. Se desarrolló una serie de materiales que ayudaron a implementar la actividad y a medir la percepción del proceso de aprendizaje durante y después de las sesiones. Los resultados indican que los alumnos han participado de forma más activa en dichas sesiones, con un buen aprovechamiento y mejora de los resultados. La opinión de los alumnos coincide con una buena valoración de la actividad para mejorar el aprendizaje de estos conceptos y expresa su interés para que este tipo de metodología sea extendida a otras actividades de los primeros cursos. La experiencia es transferible a asignaturas en las que se realice una misma actividad docente con cierta periodicidad persiguiendo potenciar el trabajo autónomo y continuo.
\end{abstract}

Palabras clave: clase invertida, matemáticas en Ingeniería, metodología activa, sesiones prácticas

Abstract- In this paper, we summarize an experience in flipped learning in lab sessions for a mathematics course for Electronics Engineering Degree. We developed a set of learning materials and opinion surveys in order to monitor students' learning process and their opinion. The results confirm that the participation of students was high and was achieved with higher marks. Students' opinion agreed with a good evaluation of this learning activity, specifically to increase practice knowledge as well as to prepare final exams. The students saw positively the extension of these learning activities to other studying context even in the first academic year. The experience is transferable to courses in which the same learning activity is carried out with certain periodicity, seeking to promote autonomous and continuous work.

Keywords: flipped classroom, mathematics in Engineering, active methodology, lab sessions.

\section{INTRODUCCIÓN}

Las asignaturas de corte matemático generalmente resultan áridas para los estudiantes universitarios de cualquier área, pero en ingeniería y titulaciones de índole técnico, son fundamentales.
Lograr motivar a los estudiantes y generar en ellos un interés creciente por estas materias es el objetivo fundamental del grupo de profesores que hemos desarrollado este trabajo.

Freeman et al. (2014) presentan la eficiencia de las metodologías activas centradas en el estudiante para el aprendizaje de asignaturas de contenidos científicos, tecnológicos y matemáticos. En esta misma línea, The Conference Board of Mathematical Sciences (CBMS, 2016) hacía una llamada a las instituciones y departamentos matemáticos para que incentivasen las prácticas docentes que implican al estudiante en el proceso de su propio aprendizaje.

Benjamin et al. (2017) describen las principales metodologías docentes de aprendizaje activo en materias de contenidos matemáticos.

Entre las distintas actividades que se enmarcan en el aprendizaje activo, nosotros nos hemos decantado por el modelo flipped learning (o flip classroom o clase invertida) Milman (2012). Esta metodología pone al alumno en el centro del proceso de aprendizaje y lo convierte en el artífice de su propio aprendizaje, potenciando la participación y el trabajo autónomo del estudiante. El diseño de las actividades nos ayuda a reducir la heterogeneidad del grupo y a favorecer el trabajo continuo.

Fidalgo et al. (2017) hacen una completa revisión de experiencias de innovación educativa desarrolladas en el marco de Flip Teaching (FT), haciendo énfasis en el impacto que ésta tiene en el aprendizaje. Asimismo presentan evidencias empíricas de la validez del modelo Micro Flip Teaching (MFT) implementado en la asignatura de Fundamentos de la Programación en el primer curso del Grado de Biotecnología.

Esta metodología también se ha puesto en práctica en asignaturas de contenidos matemáticos y se han encontrado evidencias empíricas que muestran que flipped learning es una metodología que contribuye a mejorar la motivación, la actitud hacia el aprendizaje y el rendimiento académico del alumnado en secundaria.

En el ámbito universitario, los estudios y las investigaciones relacionados con las matemáticas no son tan numerosos. En 
concreto, en el entorno de las matemáticas en escuelas de ingeniería, podemos citar los trabajos de Capilla et al. (2015) y Castilla et al. (2015).

En este estudio nos hemos ayudado del Método de Aplicación de la INnovación educativa - Method for Applying Innovation in educatioN, abreviadamente MAIN, (FidalgoBlanco et al., 2018) como herramienta para definir, estructurar y planificar la innovación educativa que hemos llevado a cabo.

En la siguiente sección presentamos el contexto en el que desarrollamos la investigación educativa, para pasar a describirla, en la sección 3.

Asimismo, es también objetivo del trabajo medir la validez del modelo pedagógico puesto en práctica, por lo que se presentarán, en las últimas secciones, las herramientas utilizadas para la recogida de datos y las conclusiones que se han derivado del análisis de dichos datos.

\section{CONTEXTO}

Nuestra continua preocupación por conseguir un aprendizaje significativo que implique una participación activa, continua y autónoma del estudiante, nos lleva a repensar los modelos formativos para ir adaptándolos a las necesidades que vamos detectando en nuestros grupos de docencia.

En este contexto, presentamos un estudio del caso en la asignatura de Matemáticas II del Grado en Ingeniería Electrónica y Automática (GIEA), desarrollado en el marco de los Proyectos de Innovación Docente de la Universidad de Zaragoza.

Se trata de una experiencia de innovación educativa diseñada siguiendo las pautas definidas por MAIN. Este método nos ha servido de herramienta para planificar la experiencia de innovación educativa siguiendo las fases que éste establece (identificación del problema raíz e indicadores de mejora, selección de métodos, descripción del proceso, valoración de esfuerzos y definición de estrategias).

La asignatura Matemáticas II se imparte en el primer semestre del primer curso, por lo que el grupo de trabajo está formado fundamentalmente por estudiantes de nuevo ingreso. En los últimos Informes de la evaluación de la calidad y de los resultados del aprendizaje del Graduado en Ingeniería Electrónica y Automática de la Universidad de Zaragoza se presentan datos de los que cabe deducir que, en esta titulación, se tienen dos perfiles de estudiantes de nuevo ingreso. En el informe referente al curso académico 2017-2018 se refleja que, por un lado está el estudiante vocacional, formado por quienes acceden en la convocatoria de junio con una nota de acceso superior a 8,252 y habiendo elegido este grado en primera opción ( 78 de las 139 plazas ofertadas). El otro perfil de estudiante corresponde a aquellos para los que esta titulación no ha sido su primera elección. La baja cifra de solicitudes en junio hace que la nota de acceso en septiembre descienda hasta 5,468. Esto confiere al grupo una gran heterogeneidad, no sólo en cuanto a motivación e inquietudes de aprendizaje, sino también en lo referente al nivel de conocimientos previos. Como se prueba en el Informe de la evaluación de la calidad del grado existe una alta correlación entre la tasa de abandono en el primer año y si esta titulación no la ha elegido el estudiante como primera opción.
Estas particularidades del alumnado tienen un impacto negativo en el rendimiento obtenido en el aprendizaje ya que, a esta heterogeneidad del grupo, se le une la pasividad observada en el proceso de aprendizaje. Pasividad que se ve acentuada en las sesiones de prácticas de laboratorio de la asignatura.

En esta asignatura de Matemáticas II, se realizan seis sesiones de ordenador (en semanas alternas) utilizando un software de cálculo simbólico. Éstas tienen un carácter formativo muy importante y resultan un complemento esencial para las sesiones presenciales de aula. El problema detectado en estas sesiones es consecuencia de la heterogeneidad del grupo que implica necesidades de tiempos muy dispares para la realización de las actividades propuestas en las sesiones de laboratorio, pues cada estudiante requiere un tiempo de asimilación y aprendizaje diferente. Esto se ve acrecentado por la falta de trabajo autónomo y continuo, ya que se suele posponer todo el estudio a los días previos a la prueba de evaluación, hecho que provoca un deficiente aprovechamiento de las prácticas.

En este contexto, nos planteamos poner en marcha una experiencia de innovación educativa con la que buscamos corregir nuestro problema raíz, esto es, conseguir una mayor homogeneidad en los subgrupos de prácticas (en cuanto al nivel de conocimientos previos) y mejorar el aprovechamiento de las sesiones de ordenador, potenciando el trabajo autónomo y continuo.

En una primera fase analizamos la situación de aprendizaje a mejorar, el perfil del estudiante y el impacto negativo que se produce. Asimismo, definimos indicadores que nos permiten medir el impacto de la nueva metodología y nos conducirán a concluir si la nueva metodología docente resuelve o mejora el problema.

En este contexto, nos hemos planteado como indicadores la participación del alumnado en las actividades propuestas y el rendimiento obtenido en las sesiones prácticas. De manera que se considerará que la nueva metodología docente resuelve o mejora el problema raíz si se consigue una mayor participación en las actividades relacionadas con las sesiones prácticas. Otro indicador que nos ha interesado controlar ha sido el tiempo dedicado por el alumno a la preparación de las actividades relativas a las sesiones prácticas. Éste nos marca la existencia de trabajo continuo a la vez que nos indica si pudiéramos estar sobrecargando al estudiante de trabajo.

En la tercera fase de MAIN vamos a describir el método de innovación educativa, presentando la tecnología utilizada, el tipo de contenidos desarrollados, las actividades diseñadas por el profesorado para ser realizadas por los alumnos, así como los cuestionarios creados para recoger información, tanto cuantitativa como cualitativa, que nos van a permitir medir la validez y alcance de la experiencia.

\section{DESCRIPCIÓN}

La metodología que se ha planteado para las sesiones prácticas combina la presencialidad con el trabajo no presencial y en ellas se hace uso de las NTIC y herramientas virtuales consiguiendo un aprendizaje mixto. De manera que, en cada sesión de prácticas se ha de realizar previamente un trabajo autónomo no presencial (estructurado según el marco de la metodología de enseñanza invertida) y un trabajo grupal 
presencial en el aula (dirigido por los profesores). Para cada sesión de prácticas se pautan las tareas a realizar por el alumno según el siguiente esquema:

Trabajo autónomo (antes de cada sesión de prácticas):

- Realización de actividades de forma individual en la que el alumno trabaje determinados conceptos relacionados con la práctica a partir de un material adicional facilitado por los profesores.

- Asimismo, el estudiante debe contestar un Cuestionario en Moodle sobre los contenidos trabajados de forma autónoma.

Trabajo grupal (durante cada sesión de prácticas):

- Revisión de las cuestiones que hayan presentado mayor dificultad en la fase de clase invertida, enfatizando en aspectos esenciales que pueden haber pasado por alto los estudiantes.

- Desarrollo de las actividades de la práctica, haciendo especial hincapié en los puntos en los que los alumnos han tenido más dificultades en la fase de autoaprendizaje.

La plataforma de Moodle resulta fundamental para activar la metodología de clase invertida pues permite albergar los materiales del curso y crear cuestionarios de evaluación. Destacar que los cuestionarios de Moodle proporcionan información que sirve de indicador, tanto a los alumnos como a los profesores, de la calidad del autoaprendizaje realizado.

Además del material docente de apoyo para la realización de las clases invertidas, cuestionarios y demás actividades relacionadas, se ha diseñado un protocolo de recogida de información para medir la validez de la metodología docente. Se registran datos de carácter cuantitativo asociados a los dos tipos de tareas (participación, tiempo dedicado al trabajo autónomo, resultados de los cuestionarios, pregunta menos veces acertada, errores frecuentes, resultados finales,...) así como el diseño e implementación de breves cuestionarios que nos han permitido adquirir datos cualitativos de la experiencia de clase invertida (nivel de satisfacción con la metodología, autopercepción del trabajo autónomo realizado,...).

En esta experiencia hemos creado cinco cuestionarios (uno por cada una de las cinco últimas sesiones de prácticas), todos respondiendo a un mismo patrón. En cada uno de éstos se planteaban 4 preguntas relativas a la evaluación de la actividad (siempre las mismas) y 5 preguntas relacionadas con los contenidos de las prácticas. De éstas últimas, las 4 primeras versaban sobre los contenidos trabajados en la sesión presencial anterior, mientras que la última hacía referencia al material que el alumno debía de trabajar de forma autónoma fuera del aula para preparar la siguiente práctica (que hemos denominado cuestión flipped).

Las cuatro cuestiones de valoración de la actividad hacían referencia al tiempo dedicado a preparar el test, la mejora de la forma de trabajar en el aprendizaje de los conceptos, la percepción de dificultad del test relativo a los ejercicios realizados en las prácticas y el concepto o conceptos más difíciles de asimilar. Esta última cuestión ha sido utilizada como retroalimentación para poder reforzar los aprendizajes que les han resultado más complicados.
Se diseñó además, un cuestionario con 6 preguntas para ser contestadas en una escala de Likert de 5 puntos, todas redactadas en la misma dirección de opinión, donde 1 era totalmente desacuerdo y 5 era totalmente de acuerdo. Los contenidos de las afirmaciones hacían referencia al trabajo autónomo, a la asimilación de los contenidos de prácticas, la capacidad para terminar todas las tareas propuestas, la capacidad para formalizar o resolver dudas, la mejora en la preparación de los exámenes finales y su opinión sobre la implantación de clase inversa en más ámbitos de primer curso.

El cuestionario ha sido completado al finalizar el segundo cuatrimestre, para que el alumno haya tenido la oportunidad de realizar prácticas similares en otras asignaturas, pero sin utilizar la metodología de clase invertida.

En la actualidad estamos realizando un análisis exhaustivo de los datos recopilados con objeto de obtener una información que nos permita medir el impacto de la innovación docente y nos guíe en la mejora de la misma. Asimismo es nuestra intención continuar trabajando en esta línea identificando la validez de los indicadores de mejora utilizados, medir la eficacia y la eficiencia de la metodología propuesta, analizando la sostenibilidad y transferibilidad de la misma.

\section{Resultados}

La participación de los alumnos se puede considerar alta. De un total de 122 alumnos matriculados en actas, 116 contestaron algún cuestionario de las 5 sesiones posibles. Por sesiones, la participación osciló entre el 67,2\% y el 87,7\%. En la figura 1, podemos ver que la cuestión flipped tiene una tasa de respuesta algo menor que la media del resto de preguntas, pero no son muy diferentes

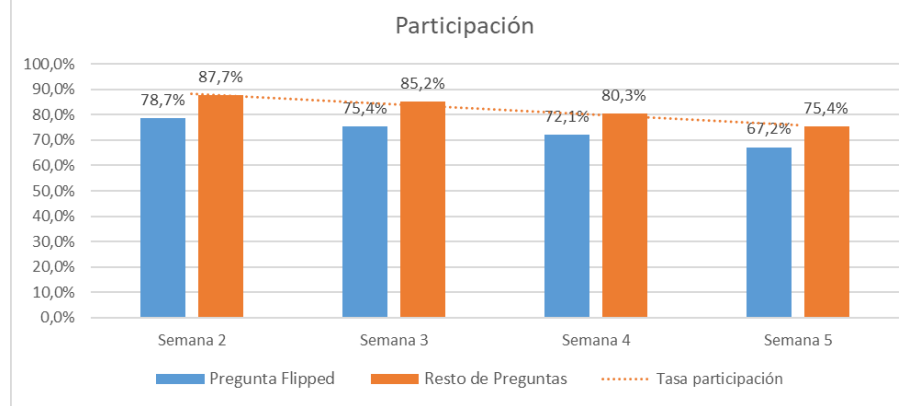

Figura 1. Tasa de respuesta a lo largo de las semanas, separadas por cuestión flipped y resto.

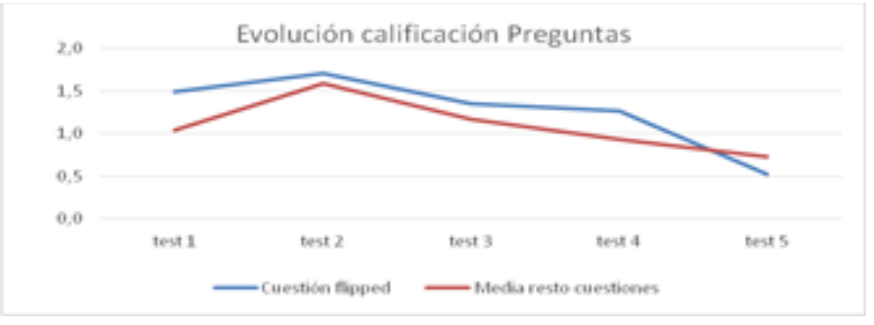

Figura 2. Evolución de las calificaciones de prácticas

En la figura 2, podemos ver las calificaciones obtenidas en las sesiones prácticas. Todas las cuestiones de opción mútiple planteadas han sido evaluadas con 2 puntos el acierto, - 0,4 puntos el fallo y 0 puntos si se dejaba en blanco. Cabe 
destacar que se han mantenido a unos buenos niveles hasta la última sesión que han empeorado. En todas las sesiones (salvo en la última) los resultados en las cuestiones que hacían referencia a contenidos trabajados desde la metodología de clase invertida han sido ligeramente mejores que el resto de cuestiones.

Respecto al tiempo dedicado a preparar los cuestionarios de prácticas y las actividades de la clase invertida de forma autónoma, los alumnos indican una media en torno a 2 horas, siendo el intervalo de una a dos horas el periodo más frecuente a lo largo de todo el curso (ver figura 3). En las dos últimas sesiones se aprecia un aumento del porcentaje de alumnos que bajan su dedicación a un tramo de menos de una hora e incluso menos de media hora.

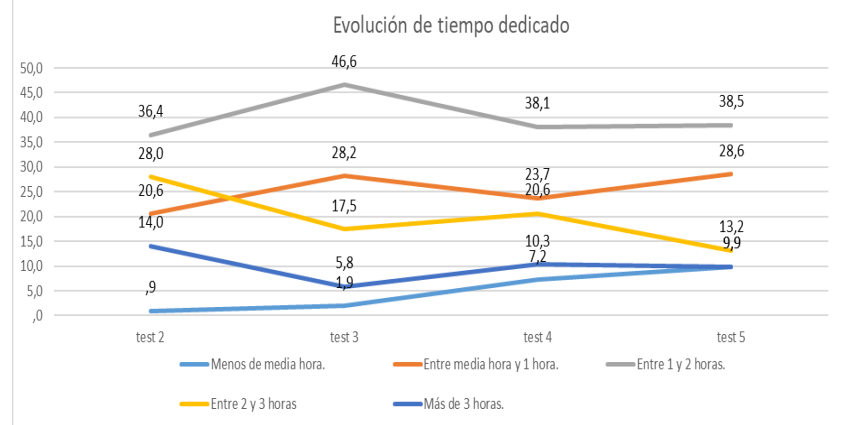

Figura 3. Evolución del tiempo dedicado a preparar las pruebas y actividades de clase invertida.

La cuestión sobre si la actividad mejora el aprendizaje, ha evolucionado de una forma poco clara (ver figura 4). Las opciones más positivas han perdido peso respecto a posiciones neutras o ligeramente negativas. Se puede deber a la percepción de mayor dificultad con las semanas y al hecho de que el alumno opine de forma conjunta sobre la actividad de clase invertida y el resto de preguntas.

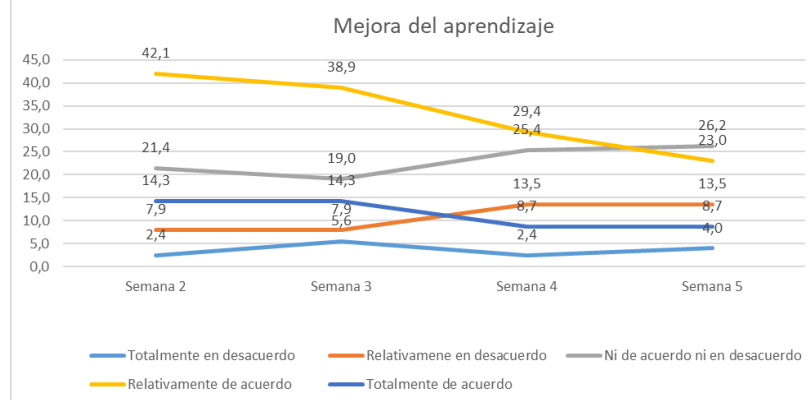

Figura 4. Evolución de la percepción de mejora del aprendizaje asociada a la actividad de clase Invertida.

En la figura 5, se muestran valores medios de la nota de prácticas en cada semana, que indican que en los dos niveles de mayor dedicación los resultados en las calificaciones son aceptables y que si la dedicación está entre media hora y dos horas comienzan a empeorar, siendo muy malos si la dedicación desciende por debajo de media hora.

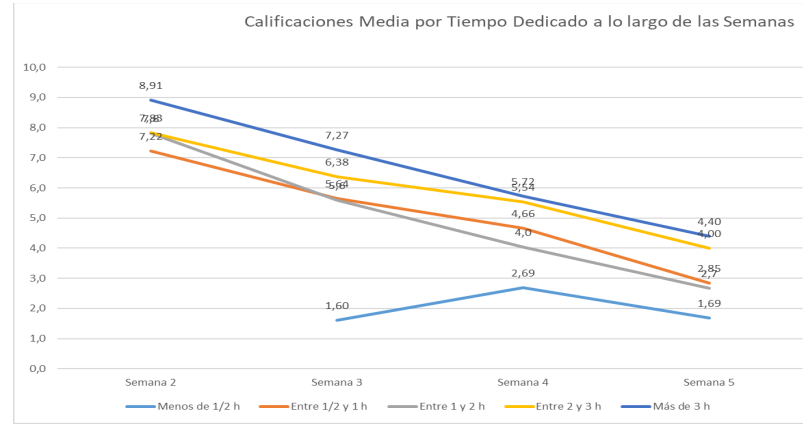

Figura 5. Evolución semanal de las calificaciones de prácticas por nivel de dedicación manifestado por los estudiantes.

En cuanto al último cuestionario, transcurrido un cuatrimestre, se consiguió la respuesta de 45 alumnos, un $37 \%$ de los posibles.

Se analizó la fiabilidad del mismo mediante el cálculo del alpha de Cronbach de 0,882 que se puede considerar muy alto, por lo que el instrumento de medida puede valorarse como de alta fiabilidad.

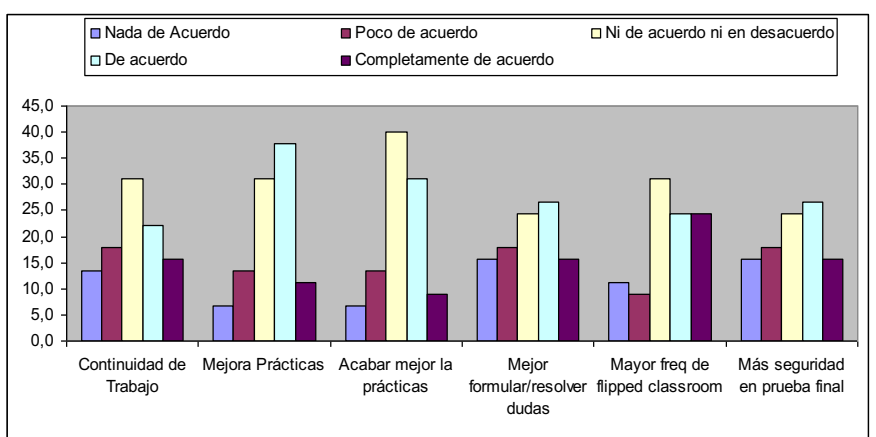

Figura 6. Distribución de valoraciones de los items del cuestionario de opinión al cuatrimestre siguiente.

En la figura 6 se puede ver la distribución de valoraciones de los 6 items que componen el cuestionario. Destaca que la valoración intermedia es en general la más frecuente, aunque en tres de ellas es superada por una valoración positiva. En concreto se valora de forma positiva la actividad de clase invertida en relación con el aprovechamiento obtenido en las prácticas, en la capacidad para formular dudas o poderlas resolver por ellos mismos y en la adquisición de mayor nivel de seguridad para enfrentarse a la prueba final.

También destaca que casi la mitad de los alumnos están de acuerdo o totalmente de acuerdo con la implantación de actividades de clase invertida en otras asignaturas de primer curso (ver Tabla 1).

La mejor asimilación de las prácticas era desde el punto de vista docente una de las principales prioridades cuando nos planteamos introducir esta innovación. El deseo de que se implante en más ámbitos, nos hace ser optimistas y pensar que con mayor presencia de actividades de tipo clase invertida, el alumno comprenda mejor las ventajas de la misma y sean capaces de aprovecharlas de una forma más eficiente. 
Tabla 1. Distribución de frecuencias del cuestionario de Opinión

\begin{tabular}{|lrrrrrrr|}
\hline Opinión & $\begin{array}{r}\text { Continuidad de } \\
\text { Trabajo }\end{array}$ & $\begin{array}{r}\text { Mejora } \\
\text { Prátticas }\end{array}$ & $\begin{array}{c}\text { Acabar mejor } \\
\text { la prócticas }\end{array}$ & $\begin{array}{r}\text { Mejor formular o } \\
\text { resolver dudas }\end{array}$ & $\begin{array}{r}\text { Mayor freq de } \\
\text { fliped classroom }\end{array}$ & $\begin{array}{c}\text { Más seguridad } \\
\text { en prueba final }\end{array}$ \\
\hline Nada de Acuerdo & 13,3 & 6,7 & 6,7 & 4,4 & 11,1 & 15,6 \\
Poco de acuerdo & 17,8 & 13,3 & 13,3 & 24,4 & 8,9 & 17,8 \\
$\begin{array}{l}\text { Ni de acuerdo ni en } \\
\text { desacuerdo }\end{array}$ & 31,1 & 31,1 & 40,0 & 31,1 & 31,1 & 24,4 \\
$\begin{array}{l}\text { De acuerdo } \\
\text { Completamente de } \\
\text { acuerdo }\end{array}$ & 22,2 & 37,8 & 31,1 & 26,7 & 24,4 & 26,7 \\
\hline Total & 15,6 & 11,1 & 8,9 & 13,3 & 24,4 & 15,6 \\
\hline
\end{tabular}

\section{CONCLUSIONES}

Esta experiencia ha exigido al profesorado un importante esfuerzo inicial en cuanto al diseño y creación de los materiales utilizados. Sin embargo, es una experiencia eficiente en cuanto a la dedicación requerida, y es de esperar una reducción considerable cuando se ponga en práctica esta misma metodología en los próximos cursos.

Los datos recopilados nos permitirán medir el esfuerzo realizado por un alumno medio y reajustar, si se considera necesario, las actividades para conseguir una metodología eficiente.

Cabe destacar que esta experiencia es sostenible en el tiempo pues no es necesario realizar ninguna inversión extra y ya se ha generado un material básico con el que continuar desarrollando esta metodología; si bien, en los próximos cursos, sería interesante continuar ampliando el banco de preguntas y revisar los materiales actuales para ir puliendo aquellos puntos en los que se observan mayores dificultades por parte de los alumnos.

Dentro de la propia asignatura, y a la vista de los resultados obtenidos en las encuestas de valoración respondidas por los alumnos en el segundo cuatrimestre, estamos estudiando la posibilidad de extender la metodología de clase invertida a otras actividades educativas en esta misma asignatura. En esta línea, nos planteamos estudiar la forma de desarrollar uno de los trabajos dirigidos aplicando dicha metodología docente. En función de los resultados que se obtengan, se analizará la idoneidad de seguir avanzando en esta dirección.

Por otra parte, esta experiencia es directamente transferible a otras asignaturas del área de Matemáticas del resto de titulaciones en las que también existen sesiones prácticas con similares problemáticas. De hecho ya se ha realizado una adaptación de experiencia en la asignatura de Matemáticas del Grado de Ingeniería de Diseño y Desarrollo de Producto. Igualmente, la experiencia, en cuanto a la aplicación de la metodología de clase invertida, puede ser transferida a asignaturas de otras áreas en las que se realicen actividades (de carácter teórico-práctico) periódicas, espaciadas entre dos y cuatro semanas en las que se desee mejorar la continuidad del trabajo del alumno en los periodos entre cada tarea, controlando que esto no suponga un sobreesfuerzo excesivo. Por otra parte, entre la valores de la metodología de clase invertida está el aprendizaje autónomo, por lo que esta metodología de clase también resulta transferible a materias en las que se persiga potenciar el trabajo autónomo.

Por último es nuestra intención continuar trabajando en la misma línea definida por esta experiencia, poniéndola en práctica de nuevo en esta misma asignatura y adaptándola a otras, con objeto de ampliar nuestro estudio y realizar contraste de resultados para tratar de definir métodos cuasiexperimentales.

\section{AgRADECIMIENTOS}

Esta investigación ha sido financiada por la universidad de Zaragoza a través de los proyectos de la convocatoria de innovación docente PIIDUZ 2018-19 (18_135).

\section{REFERENCIAS}

Benjamin, B., Bremser, P., Duval, A.M., Lockwood, E. y White, D., (2017) What Does Active Learning Mean For Mathematicians? Notices of the AMS, 64(2),124-129, doi:10.1090/noti1472

Capilla, M.T., García, B., Moll, S., Moraño, J.A., Roselló, M.D. y Sánchez-Ruiz, L.M. (Junio 2015). Clase inversa en prácticas informáticas de Matemáticas - Ingeniería Aeroespacial. Congreso In-Red 2015 Universitat Politècnica de València, doi:10.4995/INRED2015.2015.1597

Castilla, G., A. Alriols, J. , G. Romana, M. y Escribano, J.J., (Julio 2015), Resultados del estudio experimental de flipped learning en el ámbito de la enseñanaza de matemáticas en ingeniería. XII Jornadas Internacionales de Innovación Universitaria Educar para transformar: Aprendizaje experiencial. Universidad Europea. Recuperado de https://abacus.universidadeuropea.es/ bitstream/handle/11268/4491/jiiu_2015_96.pdf?sequenc $\mathrm{e}=2$ \&isAllowed $=\mathrm{y}$

Conference Board of the Mathematical Sciences (2016) Active Learning in Post-Secondary Mathematics Education. www.cbmsweb.org/Statements/Active_Learning_Statem ent.pdf

Fidalgo-Blanco, A., Martínez-Núñez, M., \& Borrás-Gene, O. (2017) Micro flip-teaching - An innovative model to promote the active involvement of students. Computers in Human Bahavior, 72, 713-723, doi: 10.1016/j.chb.2016.07.060

Fidalgo-Blanco, Á., Sein-Echaluce Lacleta, M. L., \& GarcíaPeñalvo, F. J. (2018). Method for Applying Innovation in educatioN (MAIN). (Technical Report GRIAL-TR2018-008). Retrieved from https://goo.gl/y99KnQ. Salamanca, Spain: Grupo GRIAL. doi:10.5281/zenodo. 1439134

Freeman, S., Eddy, S., McDonough, M. , Smith, M., Okoroafor, N., Jordt, H. y Wenderoth, M.P. ( Junio 2104) Active learning increases student performance in science, engineering, and mathematics, Proceedings of the National Academy of Sciences, 111 (23) 8410-8415; doi:10.1073/pnas.1319030111

Informe de evaluación de la calidad y resultados del aprendizaje del Graduado en Ingeniería Electrónica y Automática de la Universidad de Zaragoza (Curso 20172018). http://zaguan.unizar.es/record/79441?ln=en

Milman, N. B. (2012). The flipped classroom strategy: What is it and how can it best be used?. Distance Learning, 9(3), 85

Kadry, S. \& El Hami, A. (2014). Flipped classroom model in Calculus II. Education, 4(4), 103- 107 
Voronina, M. V., Moroz, O. N., Sudarikov, A. E., Rakhimzhanova, M. B. y Muratbakeev, E. K. (2017). Systematic Review and Results of the Experiment of a Flipped Learning Model for the Courses of Descriptive
Geometry, Engineering and Computer Graphics, Computer Geometry. Eurasia Journal of Mathematics, Science and Technology Education, 13(8), 4831-4845. doi: 10.12973/eurasia.2017.00967a 